\title{
Roi Livne (2019) Values at the End of Life. The Logic of Palliative Care
}

\author{
Harvard University Press, Cambridge, Massachusetts, 341 Seiten, \\ 41,00€, ISBN-13: 978-0-674-54517-5; €41
}

\section{Christoph Rehmann-Sutter}

Angenommen: 8. Juni 2021 / Online publiziert: 29. Juni 2021

(C) Der/die Autor(en) 2021

Roi Livnes Buch zur neuen Ökonomie des Sterbens verspricht nichts Erbauliches. Er will aufdecken, weshalb die Hospiz- und Palliativbewegung in den USA im Grunde eine grandiose Sparübung eines immer teurer werdenden Gesundheitssystems ist. Die Beschwörung der Autonomie der Behandelten und die sorgfältige Respektierung ihres Willens am Lebensende diene dazu, den Verzicht auf Behandlungen zu rechtfertigen, die eigentlich nur aus Kostengründen ein Problem darstellen.

Mit ,The Logic of Palliative Care“, wie der Untertitel des Buches lautet, ist genau das gemeint: Die Palliativmedizin löst mit Hilfe des Patient:innenwillens ein gesellschaftliches Problem - indem sie die Patient:innen dazu bringt, teure Behandlungen selbstbestimmt abzulehnen. Die Logik der Palliativmedizin sei es, Patient:innen mittels empathischer Gespräche in einen rekursiven Prozess hineinzumanövrieren, ,in which statements that patients made could be gradually transformed into an embrace of economized dying“" (S. 242).

Um ein Bild der Medizin am Lebensende zu zeichnen, benutzte der Soziologe Livne historisches Material zur Entwicklung des Hospiz- und Palliativwesens und führte als freiwilliger Palliativhelfer eine Reihe von teilnehmenden Beobachtungen in drei kalifornischen Palliativstationen durch.

Das erste Kapitel beschreibt die Entwicklung des Hospizwesens und der Palliativmedizin in den USA, seit 1974 in New Haven, Connecticut der erste Hospizpatient aufgenommen wurde. In den darauffolgenden vierzig Jahren wurden immer mehr Hospize gegründet und Palliativstationen eingerichtet, bis nach Angaben der National Hospice and Palliative Care Organization 2017 44,6\% aller Sterbefälle in einem Hospizprogramm stattfanden. Livne unterstellt dieser Bewegung eine Nützlichkeit, die sich in einem speziellen ,palliativmedizinischen Blick“ ausdrücke.

Prof. Dr. Christoph Rehmann-Sutter $(\bowtie)$

Universität zu Lübeck, Lübeck, Deutschland

E-Mail: christoph.rehmannsutter@uni-luebeck.de 
Die Strategie hinter der Stärkung des Patient:innenwillens, etwa durch „DNR orders“, ist für Livne eindeutig: Wenn es gelingt, die Patient:innen mehr in die Entscheidungen über ihre Behandlungen am Lebensende einzubinden, läuft es am Ende darauf hinaus, dass viele Behandlungen abgelehnt werden, die sonst noch stattgefunden hätten. Die Palliativmedizin wird so als mächtige Bewegung in der Medizin dargestellt, die durch die Art ihrer Information und durch die Techniken der Gesprächsführung wesentlich darauf Einfluss nehmen kann, was die Patient:innen wünschen.

Das zweite Kapitel stellt die Triebkräfte der Ökonomisierung dar: 1. Industrialisierung des Hospizwesens; 2. ökonomische Definition des Lebensendes; 3. Transformation des Krankenhausmarktes, die die Häuser unter Druck setzte und dazu zwang, Geschäftsstrategien zu finden, um überhaupt solvent zu bleiben; 4. die Palliativbewegung selbst, die die Krankenhausadministrationen davon überzeugte, dass die Einrichtung von Palliativstationen und die Ermächtigung der Palliativmedizin zu Einsparungen führen.

Im dritten Kapitel widerspricht Livne der verbreiteten Ansicht, dass Patient:innen, wenn man sie selbst bestimmen lässt, die meisten aggressiven lebenserhaltenden Therapien ablehnen würden. Dieses Bild einer Win-Win-Situation (,,weniger ist besser") stimme mit aktuellen Erhebungen nicht überein, die zeigen würden, dass Menschen, die näher an ihr Lebensende kommen, mehr Interesse an lebenserhaltenden und -verlängernden Behandlungen haben. Die Ökonomisierung des Sterbens musste also, so die These Livnes, gegen Widerstand durchgesetzt werden.

Das kontroverseste Kapitel scheint mir das vierte (,Making the Dying Subject“). Darin geht es um die Subjektivierung der Patient:innen-Wünsche. Man klärt heute die Behandelten über ihre Behandlungen auf und lässt sie möglichst selbst entscheiden. Das empfahl die Bioethik mit Nachdruck, weil es die Patient:innen als Personen ernst nimmt, ihre Rechte auf Selbstbestimmung respektiert und die Care verbessert. Die dazu untersuchten Interviewausschnitte enthalten aber mehr Ebenen als die einfache Botschaft, die Livne aus ihnen zieht. Es wird deutlich, wie voreingenommen Livne sein Material liest. Die Ablehnung einer Operation bedeutet für ihn immer eine Einsparung. Für eine aufgeklärte Patientin mit einer Tumorerkrankung im fortgeschrittenen Stadium kann diese Operation aber durchaus auch eine Belastung bedeuten, die sie dann aus guten Gründen ablehnen kann, selbst wenn sie ihr noch Lebenszeit verspricht. Livne unterstellt, ohne es zu begründen, dass es meistens schlecht für die Patientin ist, eine medizinisch noch mögliche Behandlung abzulehnen.

Das fünfte Kapitel („Goat Taming“) will Techniken darstellen, die es den Palliativmediziner:innen ermöglichen, den Patient:innenwillen nach ihrem Gutdünken zu manipulieren. Kliniker:innen würden die Autonomie stark beeinflussen (,heavily influence“, S. 242). Sie würden die Patient:innen in einen rekursiven Verhandlungsprozess einbinden, in dem ihr Wille graduell verändert werde, bis sie selbst zu sparen wünschen (,transformed into an embrace of economized dying“, S. 242).

Vieles in diesem Buch klingt beinahe nach einer Verschwörungstheorie. Die Palliativmedizin strebe zu einer umfassenden Macht im Kliniksystem und mache die Patient:innen (d.h. uns alle) nur glauben, dass sie von ihr empathischer gepflegt und in ihrem Willen respektiert werden. Aber eigentlich gehe es ihr darum, sie gefügig 
zu machen, damit sie selbst in eine sparsamere Medizin einwilligen, die ihnen dann beim Sterben Lebenszeit wegnimmt.

Trotz aller Vorbehalte ließ mich das Buch auch nachdenklich zurück. Man muss sich fragen: Was wäre, wenn es - z. B. in den USA, wo Millionen Menschen ohne oder mit unzureichendem Versicherungsschutz dastehen - doch zumindest zum Teil stimmt? Ein ökonomischer Druck, Menschen auf die Palliativschiene zu lenken, um ihnen immerhin irgendeine Medizin zu bieten, die ihre Wünsche respektiert und ihre Symptome behandelt, könnte unter solchen Bedingungen durchaus real sein.

Funding Open Access funding enabled and organized by Projekt DEAL.

Open Access Dieser Artikel wird unter der Creative Commons Namensnennung 4.0 International Lizenz veröffentlicht, welche die Nutzung, Vervielfältigung, Bearbeitung, Verbreitung und Wiedergabe in jeglichem Medium und Format erlaubt, sofern Sie den/die ursprünglichen Autor(en) und die Quelle ordnungsgemäß nennen, einen Link zur Creative Commons Lizenz beifügen und angeben, ob Änderungen vorgenommen wurden.

Die in diesem Artikel enthaltenen Bilder und sonstiges Drittmaterial unterliegen ebenfalls der genannten Creative Commons Lizenz, sofern sich aus der Abbildungslegende nichts anderes ergibt. Sofern das betreffende Material nicht unter der genannten Creative Commons Lizenz steht und die betreffende Handlung nicht nach gesetzlichen Vorschriften erlaubt ist, ist für die oben aufgeführten Weiterverwendungen des Materials die Einwilligung des jeweiligen Rechteinhabers einzuholen.

Weitere Details zur Lizenz entnehmen Sie bitte der Lizenzinformation auf http://creativecommons.org/ licenses/by/4.0/deed.de.

Interessenkonflikt C. Rehmann-Sutter gibt an, dass kein Interessenkonflikt besteht. 